\title{
Evaluation of moxifloxacin for the treatment of tuberculosis: 3 years of experience
}

\author{
A.D. Pranger*, R. van Altena ${ }^{\#}$, R.E. Aarnoutse ", D. van Soolingen ${ }^{+, \S}$, D.R.A. Uges*, \\ J.G.W. Kosterink*, T.S. van der Werf ${ }^{f}$ and J.W.C. Alffenaar*
}

ABSTRACT: Moxifloxacin (MFX) is a powerful second-line anti-tuberculosis (TB) agent, but the optimal dose has not yet been established and long-term safety data are scarce.

We retrospectively reviewed the medical charts of TB patients treated at the Tuberculosis Centre Beatrixoord, University Medical Centre Groningen (Haren, the Netherlands) receiving MFX $400 \mathrm{mg}$ once daily as part of their TB treatment between January 12006 and January 1 2009. Safety data and drug-drug interactions were evaluated. Efficacy was predicted based on the area under the concentration-time curve up to $24 \mathrm{~h}$ post-dosage $\left(\mathrm{AUC}_{0-24 \mathrm{~h}}\right) /$ minimal inhibitory concentration (MIC) ratio.

89 patients were treated with a median dose of $6.9 \mathrm{mg} \cdot \mathrm{kg}^{-1} \mathrm{MFX}$ once daily for a median period of 74 days. Discontinuation of therapy occurred in only three patients due to gastrointestinal sideeffects and hypersensitivity. Pharmacokinetic analysis showed an $\mathrm{AUC}_{0-24 \mathrm{~h}} / \mathrm{MIC}$ ratio $<100$ in eight out of 16 patients. A large variation in protein binding affected the unbound $A U C_{0-24 h}$ considerably.

These data show that MFX treatment was well tolerated in 89 patients receiving a dose of $400 \mathrm{mg}$ once daily for a prolonged period. Considering the variability in (un)bound $\mathrm{AUC}_{0-24 \mathrm{~h}} / \mathrm{MIC}$ ratio, therapeutic drug monitoring is recommended in selected patients (i.e. rifampicin comedication; MIC $\geqslant 0.25 \mathrm{mg} \cdot \mathrm{L}^{-1}$ ) to assess optimal therapy.

KEYWORDS: Moxifloxacin, pharmacokinetics, rifampicin, safety, tuberculosis

M oxifloxacin (MFX), a fluoroquinolone with an in vitro and in vivo bactericidal activity against Mycobacterium tuberculosis, is used for the treatment of multidrugresistant (MDR) tuberculosis (TB) or in the case of intolerance to first-line TB agents and is presently under evaluation for its potential to shorten TB treatment [1]. In addition, MFX seems useful in the case of resistance against early generation fluoroquinolones [2]. Although MFX has widely been prescribed for the treatment of $\mathrm{TB}$, one should keep in mind that the drug is not labelled for this indication [3] and there is paucity of data on the optimal dose and safety/tolerability of treatment durations longer than 2 weeks of the current regimen of $400 \mathrm{mg}$ MFX once daily.

As for other fluoroquinolones, the area under the plasma concentration-time curve (AUC) relative to the minimal inhibitory concentration (MIC) has been suggested as the best parameter to predict in vivo efficacy against Gram-negative bacteria and M. tuberculosis [4-6]. Modelling studies suggest that a daily dose of $600-800 \mathrm{mg}$ MFX should be considered for optimal killing of the bacteria and to obtain a probability of $86-93 \%$ of reaching the target associated with suppression of drug-resistant mutants (i.e. unbound area under the concentration-time curve up to $24 \mathrm{~h}$ post-dosage $\left(\mathrm{AUC}_{0-24 \mathrm{~h}}\right) /$ MIC ratio 53) [7], which is higher than the currently used dose of $400 \mathrm{mg}$ once daily. As the efficacy of the treatment is determined by the protein-unbound (free) concentration, the MFX protein binding should also be taken into account [5].

The clinically most relevant drug interaction in TB patients is that of MFX and rifampicin (RIF), resulting in a predicted decrease of MFX exposure of $31 \%$ [8, 9]. Mineral supplements such as iron and zinc, or antacids might decrease the bioavailability of MFX as well [10], but after a daily dose of $400 \mathrm{mg}$ MFX in combination with food or calcium supplements, the MFX AUC is not significantly affected [10].

The major concern for prolonged treatment is that adverse effects may result in decreased compliance, potentially resulting in drug resistance. The adverse effects of MFX, such as vomiting and diarrhoea [10], could influence the tolerability of MFX during prolonged treatment. A potential serious but infrequent adverse effect of MFX is QT prolongation [11].
AFFILIATIONS

Depts of *Hospital and Clinical

Pharmacy, and

fInternal Medicine and Pulmonary

Diseases and Tuberculosis,

University Medical Center Groningen, University of Groningen, Groningen,

\#Tuberculosis Centre Beatrixoord,

University Medical Center Groningen, University of Groningen, Haren,

Depts of "Clinical Pharmacy, and

${ }^{\text {s}}$ Pulmonary Diseases and Medical

Microbiology, Radboud University

Nijmegen Medical Centre, Nijmegen, and

${ }^{+}$National Tuberculosis Reference Laboratory, National Institute for

Public Health and the Environment (RIVM), Bilthoven, The Netherlands.

CORRESPONDENCE

J.W.C. Alffenaar

Dept of Hospital and Clinical

Pharmacy, University Medical

Center Groningen

University of Groningen

P0 box 30.001

9700 RB Groningen

The Netherlands

E-mail: j.w.c.alffenaar@umcg.nl

Received:

Nov 152010

Accepted after revision:

Jan 312011

First published online:

Feb 102011

European Respiratory Journal

Print ISSN 0903-1936

Online ISSN 1399-3003 
MFX $400 \mathrm{mg}$ once a day is safe and well tolerated during prolonged treatment in studies with a small number of patients $[12,13]$. Despite increasing experience with MFX in TB patients [14-16], larger studies are needed to confirm efficacy and longterm safety of an adequate dosage. Safety data to support switching to the suggested higher dose is scarce $[11,17,18]$.

The objective of this study was to evaluate pharmacokinetic and pharmacodynamic parameters, drug-drug interactions and safety/tolerability of MFX in TB treatment retrospectively in order to assess if optimal therapy has been given. As a result, these findings will contribute to dose finding and enhance the knowledge of pharmacokinetics of MFX in future TB patients.

\section{PATIENTS AND METHODS}

A retrospective chart review was performed for all patients receiving MFX (Avelox®; Bayer, Leverkusen, Germany) for at least 5 days (steady state) as part of their TB treatment [19] at the Tuberculosis Centre Beatrixoord, University Medical Center Groningen (Haren, the Netherlands) between January 12006 and January 1 2009. Demographic and medical data were collected from the medical chart including age, sex, weight, height, ethnicity, comorbidity, diagnosis, localisation of TB, MIC, resistance pattern, medical history, dose and duration of MFX treatment, dose and duration of (TB) co-medication and MFX-induced adverse effects. According to the retrospective nature of this study, approval by our local ethical committee was not required.

\section{Pharmacokinetics and pharmacodynamics}

When available, MFX concentration in plasma and plasma ultrafiltrate (20 $\mathrm{min}$ at room temperature, $1,640 \times \mathrm{g}$ in a fixed angle rotor, Hettich EBA 21; Andreas Hettich GmbH and Co.KG, Beverly, MA, USA) was determined by a validated liquid chromatography-tandem mass spectrometry analysing method [20]. Samples were eligible for evaluation when obtained at steady state, which was at least 5 days after treatment [19]. Different pharmacokinetic parameters, including $\mathrm{AUC}_{0-24 \mathrm{~h}}$ for plasma were determined with a standard one-compartmental pharmacokinetic method using the KINFIT module of MW $\backslash$ Pharm 3.60 (Mediware, Zuidhorn, the Netherlands). The $\mathrm{AUC}_{0-}$ $24 \mathrm{~h}$ was calculated according to the log-linear trapezoidal rule. As the MFX protein binding may be concentration dependent (range 0.077-0.6) [5], we chose to determine the unbound concentration in plasma ultrafiltrate for a low $\left(<1.0 \mathrm{mg} \cdot \mathrm{L}^{-1}\right)$ and a high MFX total plasma (protein bound + unbound) concentration $\left(>1.0 \mathrm{mg} \cdot \mathrm{L}^{-1}\right)$ for each individual concentration-time curve. The mean protein-unbound concentration was used to assess the unbound concentration-time curve.

The drug susceptibility test of the available $M$. tuberculosis isolates was performed with the Middlebrook 7H10 agar dilution method [21] at the Dutch National Tuberculosis Reference Laboratory (National Institute for Public Health and the Environment (RIVM), Bilthoven, the Netherlands).

Both protein-bound and -unbound $\mathrm{AUC}_{0-24 \mathrm{~h}} / \mathrm{MIC}$ ratio could be calculated and the number of patients having an $\mathrm{AUC}_{0-24 \mathrm{~h}} /$ MIC ratio $>100$ was determined. Because efficacy of treatment is determined by the protein-unbound (free) concentration, a total (i.e. bound and unbound) $\mathrm{AUC}_{0-24 \mathrm{~h}} / \mathrm{MIC}$ ratio $>100[5,22]$ is translated into an unbound $\mathrm{AUC}_{0-24 \mathrm{~h}} / \mathrm{MIC}$ ratio exceeding at least 60. This ratio stems from the most frequently reported value of protein binding of approximately $40 \%$ for MFX [23], which results in an unbound fraction of 0.6.

\section{Drug-drug interactions}

Drug-drug interactions may influence MFX efficacy by interfering with MFX absorption, metabolism or excretion. We evaluated co-medication for the following drugs: RIF, antacids, mucosal protectants, minerals (e.g. zinc and iron) and didanosine $[8,10]$.

Based on the pharmacokinetic curves of both patients with and without concomitant use of MFX and RIF, two separate onecompartmental pharmacokinetic population models with firstorder absorption without lag time were generated using the MFX dose, the body surface area of the TB patients, the serum creatinine concentration and the observed MFX plasma concentrations using an iterative two-stage Bayesian procedure (MW $\backslash$ Pharm 3.60) [24]. All pharmacokinetic curves are obtained after reaching steady-state concentrations of MFX and, instead of concomitant use of MFX and RIF, after reaching steady-state concentrations of RIF.

\section{Safety/tolerability}

To evaluate the safety of MFX treatment, all recorded adverse effects were retrieved from the medical chart, including diarrhoea, vomiting and QT prolongation. MFX is contraindicated in patients with transaminase values more than fivetimes the upper level of normal [3]. Hepatic injury was characterised if the value of at least one of the following enzymes exceeds five-times the upper level of normal during MFX treatment along with no hepatic dysfunction (i.e. five times upper level of normal) observed at baseline (grade 3 common toxicity criteria (CTC)): aspartate aminotransferase (ASAT; $>200 \mathrm{U} \cdot \mathrm{L}^{-1}$ ), alanine transaminase (ALAT; $>225 \mathrm{U} \cdot \mathrm{L}^{-1}$ ) and gamma-glutamyl transpeptidase (GGT; $>200-275 \mathrm{U} \cdot \mathrm{L}^{-1}$ ) [25]. Renal injury was defined as serum creatinine level increased $25 \%$ compared with baseline (grade 1 CTC) [25]. The upper level of normal was defined at a serum creatinine value of $112.5 \mu \mathrm{mol} \cdot \mathrm{L}^{-1}$ (females) or $137.5 \mu \mathrm{mol} \cdot \mathrm{L}^{-1}$ (males). A QT period of $>500 \mathrm{~ms}$ is associated with increased risk of cardiac events [26]. After $\sim 2$ weeks of treatment and in cases of any dose escalation of MFX, a routine three-lead ECG was obtained by a physician from each patient. Any abnormal observation on the ECG was recorded in the medication chart. To estimate the risk of QT prolongation by long-term MFX treatment, we identified risk factors that (apart from administration of MFX) can result in, or aggravate, QT prolongation in TB patients treated with MFX. The following risk factors were evaluated in patients: female sex, hepatic dysfunction, pro-arrhythmic conditions (i.e. abnormal cardiac repolarisation on baseline ECG), hypokalaemia $\left(<3.5 \mathrm{mmol} \cdot \mathrm{L}^{-1}\right.$ serum $)$, hypomagnesaemia $\left(<0.7 \mathrm{mmol} \cdot \mathrm{L}^{-1}\right.$ blood) and simultaneous treatment with anti-dysrhythmics class IA and class III, antipsychotics, tricyclic antidepressants or the antihistaminic drug terfenadine $[3,27,28]$.

To determine potential causality between adverse effects and MFX treatment, the Naranjo algorithm was used (0-9 points, of which 9 represents the highest likelihood) [29]. The correlation between total drug exposure (AUC) and adverse effects was explored. 
Special attention was paid to discontinuation of MFX. Reasons were categorised into four categories: 1) MFX was started based on expected drug resistance (country of origin, medical history) and discontinued after the drug susceptibility pattern became available and showed an isolate susceptible to first-line agents; 2) MFX was started because of intolerance to first-line TB agents and discontinued after the adverse effects had been resolved and first-line drugs were successfully re-introduced; 3) completion of MFX treatment; and 4) MFX-induced adverse events.

\section{Statistics}

When not normally distributed, nonparametric tests were used, i.e. Mann-Whitney U-test and Wilcoxon rank sum test for ordinal data, and Chi-squared tests were used for nominal data.

\begin{tabular}{lc} 
TABLE 1 Patient characteristics at baseline \\
Female & \\
Age yrs & $32(36)$ \\
Weight kg & $35(27-47)$ \\
Length cm & $58.3(49.6-66.7)$ \\
BMI kg. m $^{-2}$ & $170(162-175)$ \\
Ethnicity & $20.1(17.9-23.0)$ \\
Caucasian & \\
Asian & $29(32.6)$ \\
African & $17(19.1)$ \\
Other & $41(46.1)$ \\
Duration of hospital stay days & $2(2.3)$ \\
Tuberculosis & $62.5(35-112.3)$ \\
Localisation & \\
$\quad$ Pulmonary & \\
Extrapulmonary & $67(75.3)$ \\
Other & $29(32.6)$ \\
Diagnosis & $5(5.6)$ \\
Sputum & \\
Other,+ & $60(67.4)$ \\
Resistance pattern & $29(32.6)$ \\
Fully susceptible & \\
MDR & \\
INH resistant & $54(60.7)$ \\
INH and ethambutol resistant & $20(22.5)$ \\
Unknown & $2(2.3)$ \\
Comorbidity & $1(1.1)$ \\
Chronic pre-existent liver disease & $12(13.5)$ \\
Chronic renal dysfunction & \\
Epilepsy & $5(5.6)$ \\
Diabetes mellitus & $1(1.1)$ \\
HIV co-infection & $1(1.1)$ \\
Alcohol abuse & $10(11.2)$ \\
\hline & $10(11.2)$ \\
& $8(9.0)$ \\
\hline
\end{tabular}

Data are presented as $n$ (\%) or median (interquartile range). ${ }^{*}: \mathrm{n}=89$. BMI: body mass index; MDR: multidrug resistant; INH: isoniazid. " diagnosis based on clinical conditions, chest radiograph, histology and/or response to therapy. ${ }^{+}:$in 29 cases, diagnosis is based on clinical conditions, chest radiograph, histology and/or response to therapy; in 17 out of 29 patients the resistance pattern was determined at a later stage.

\section{RESULTS}

\section{Patient characteristics}

A retrospective chart review was performed for 89 patients with a median (interquartile range (IQR)) age of 35 (27-47) yrs; 32 $(36 \%)$ patients were female and $57(64 \%)$ were male. One patient (transgender) was excluded because of the unknown influence of administered hormones on several important clinical parameters. Pulmonary TB was the most common diagnosis (67 (75\%) patients). In 32 (36\%) patients MFX was started because of expected resistance (MDR-TB) on the basis of treatment history. Patients received MFX $400 \mathrm{mg}$ once daily, which equals a median (IQR) dose of $6.9(6.0-8.1) \mathrm{mg} \cdot \mathrm{kg}^{-1}$. Patients were treated with MFX for a median (IQR) period of 74 (29-186) days. During treatment there was a dose escalation to $800 \mathrm{mg}$ once daily in four patients. The dose was in all cases escalated to $800 \mathrm{mg}$ because of an $\mathrm{AUC}_{0-24 \mathrm{~h}} / \mathrm{MIC}$ ratio $<100$ (i.e. $\mathrm{AUC}_{0-24 \mathrm{~h}} / \mathrm{MIC} 56-$ $83)$ in combination with an $\mathrm{AUC}_{0-24 \mathrm{~h}}$ value $<50 \mathrm{mg} \cdot \mathrm{h} \cdot \mathrm{L}^{-1}(\mathrm{n}=3)$ or a low $\mathrm{AUC}_{0-24 \mathrm{~h}}\left(\right.$ i.e. $\mathrm{AUC}_{0-24 \mathrm{~h}} 24.1 \mathrm{mg} \cdot \mathrm{h} \cdot \mathrm{L}^{-1}$ ) in combination with an unknown resistance pattern at the start of therapy $(n=1)$. Thereafter, the dose was reduced to $600 \mathrm{mg}$ once daily based on an $\mathrm{AUC}_{0-24 \mathrm{~h}} / \mathrm{MIC}$ ratio $>100(\mathrm{n}=2)$ or based on resistance pattern, which was unknown at the start of therapy $(n=1)$. Two patients died from AIDS and TB, not related to MFX. An overview of the baseline patient characteristics and anti-TB drugs is shown in tables 1 and 2 .

\section{Pharmacokinetics and pharmacodynamics}

From 16 patients a full pharmacokinetic curve in plasma was available. The mean plasma concentration-time curve is shown in figure 1. For nine of these patients plasma ultrafiltrate was available. We observed an interindividual variable plasma protein binding ranging from 11.0 to $41.7 \%$. The median (IQR)

\section{TABLE 2 Anti-tuberculosis (TB) medication*}

\begin{tabular}{lc} 
First-line oral anti-TB drugs & \\
Isoniazide & $69(77.5)$ \\
Rifampicin & $68(76.4)$ \\
Pyrazinamide & $69(77.5)$ \\
Ethambutol & $65(73.0)$ \\
Rifabutin & $2(2.3)$ \\
Injectable anti-TB drugs & \\
Amikacin & $24(27.0)$ \\
Kanamycin & $16(18.0)$ \\
Fluoroquinolones & \\
$\quad$ Ofloxacin & $1(1.1)$ \\
$\quad$ Moxifloxacin & $89(100)$ \\
Oral bacteriostatic second-line & \\
anti-TB drugs & \\
Protionamide & $17(19.1)$ \\
Cycloserine & $4(4.5)$ \\
Anti-TB drugs with unclear efficacy & \\
or unclear role in MDR-TB treatment & \\
Linezolid & $22(24.7)$ \\
Clofazimine & $17(19.1)$ \\
Thioacetazon & $3(3.4)$ \\
Azithromycin & $3(3.4)$ \\
Clarithromycin & $2(2.3)$ \\
\hline
\end{tabular}

Data are presented as $n(\%){ }^{*}: \mathrm{n}=89$. MDR: multidrug resistant. 


\begin{tabular}{lccc}
\hline TABLE 5 & \multicolumn{3}{c}{$\begin{array}{l}\text { Moxifloxacin population pharmacokinetic model } \\
\text { parameter values }\end{array}$} \\
\hline Parameter & RIF & No RIF & p-value \\
\hline $\mathbf{C L ~ L} \cdot \mathbf{h}^{-\mathbf{1}} / \mathbf{1 . 8 5} \mathbf{~ m}^{\mathbf{2}}$ & $22.6 \pm 8.5$ & $15.5 \pm 7.5$ & 0.083 \\
$\mathbf{V}_{\mathbf{d}} \mathbf{~} \cdot \mathbf{k g}^{-\mathbf{1}} \mathbf{L B M c}$ & $3.46 \pm 0.32$ & $2.90 \pm 0.29$ & 0.009 \\
$\mathbf{K}_{\mathbf{a}} \mathbf{h}^{-\mathbf{1}}$ & $3.638 \pm 1.696$ & $3.227 \pm 1.423$ & 0.515 \\
$\mathbf{T}_{\text {lag }} \mathbf{h}$ & $0.55 \pm 0.15$ & $0.69 \pm 0.12$ & 0.009 \\
$\mathbf{F}$ & 1 (fixed) & 1 (fixed) & \\
\hline
\end{tabular}

Data are presented as mean $\pm S D$, unless otherwise stated. $n=16$. RIF: rifampicin; $C L$ : apparent clearance; $V_{d}$ : volume of distribution; LBMc: lean body mass; $\mathrm{K}_{\mathrm{a}}$ : absorption rate constant; $\mathrm{T}_{\text {lag }}$ : lag time; $\mathrm{F}$ : bioavailability.

16 patients had an $\mathrm{AUC}_{0-24 \mathrm{~h}} / \mathrm{MIC}$ ratio $>100$ and eight had a ratio $<100$ (range 21-83). The geometric mean unbound plasma $\mathrm{AUC}_{0-24 \mathrm{~h}}$ and unbound $\mathrm{AUC}_{0-24 \mathrm{~h}} / \mathrm{MIC}$ were equal to 22 (range $12-64$ ) $\mathrm{mg} \cdot \mathrm{h} \cdot \mathrm{L}^{-1}$ and 59 (range $16-257$ ) $\mathrm{mg} \cdot \mathrm{h} \cdot \mathrm{L}^{-1}$, respectively. In plasma ultrafiltrate, five of the nine patients had an unbound $\mathrm{AUC}_{0-24 \mathrm{~h}} / \mathrm{MIC}$ ratio $>60$ and four had a ratio $<60$ (range 16-49). Three patients had a high MIC of $1 \mathrm{mg} \cdot \mathrm{L}^{-1}$ and therefore a low unbound and total $\mathrm{AUC}_{0-24 \mathrm{~h}} / \mathrm{MIC}$ ratio.

\section{Safety/tolerability}

MFX was well tolerated; it was discontinued in only three $(3.4 \%)$ patients because of gastrointestinal adverse effects $(n=2)$ and hypersensitivity $(n=1)$. An overview of adverse effects is shown in table 4. Renal function tests did not deteriorate during treatment. We observed a significant decrease (ASAT: $p=0.004$; ALAT: $p=0.020$ ) in liver enzymes during MFX treatment. However, in one patient normal GGT values increased to more than five-times the upper level of normal (Naranjo score 3). In four patients, serum creatinine values increased during treatment, along with an increase in body weight, but remained within normal limits and this increase in serum creatinine might reflect increased muscle mass with stable renal function. Vomiting was observed in two (2\%; Naranjo score 3) and diarrhoea in eight (9\%; Naranjo score 3 or 4 ) patients. 35 patients had at least one additional risk factor for QT prolongation, 17 patients had two additional risk factors and one patient had four risk factors, but no QT prolongation was observed. In our study population, female sex was the most common potential risk factor for QT prolongation. $\mathrm{AUC}_{0-24 \mathrm{~h}}$ values could not be related to adverse events as adverse events were scarce and $\mathrm{AUC}_{0-24 \mathrm{~h}}$ values were only determined in a subset of patients.

\section{Drug-drug interactions}

RIF was frequently co-administered with MFX. In 68.5, 10.1 and $1.1 \%$ of the patients, MFX was combined with RIF in a dose of 600, 450 and $150 \mathrm{mg}$, respectively.

Full pharmacokinetic concentration-time curves were available in six patients who received MFX alone and in 10 patients who received RIF and MFX. Co-medication with RIF did not significantly reduce the plasma $A_{U C} C_{0-24 h}$ value with a geometric mean of 36.8 (range 12.7-50.4) versus 21.3 (range 8.5$72.2) \mathrm{mg} \cdot \mathrm{h} \cdot \mathrm{L}^{-1}(\mathrm{p}=0.104)$. No significant difference between
MFX dose in $\mathrm{mg} \cdot \mathrm{kg}^{-1}$ was observed between patients with or without RIF concomitant treatment of MFX ( $p=0.871)$. Population pharmacokinetic analysis (table 5) showed that the apparent clearance of MFX was (not significantly) induced in patients with concomitant use of MFX and RIF ( $p=0.083)$, but this induction was due to interpatient variability in both groups. MFX was not simultaneously administered with antacids, mucosal protectants, minerals or didanosine.

\section{DISCUSSION}

We observed a large variation in protein binding. This is an important finding as only unbound drug contributes to antimicrobial effect. Malnutrition and deterioration in clinical condition upon admission is the most plausible explanation for these large variations. However, because of the retrospective nature of this study and the relatively small sample size $(n=9)$ with a known unbound MFX concentration, we cannot confirm this hypothesis. Therefore, it seems logical to determine the unbound MFX concentration in each individual whenever facilities are available. As the fraction of unbound MFX appeared not to be concentration dependent, contrary to earlier reports [5], a single blood sample can be used to assess plasma protein binding at a specific time in treatment as plasma protein levels may vary during treatment.

The $\mathrm{AUC}_{0-24 \mathrm{~h}} / \mathrm{MIC}$ ratio is the best parameter to predict efficacy of MFX and a ratio exceeding 100 is desirable $[5,6,22]$. In eight of the 16 patients $\mathrm{AUC}_{0-24 \mathrm{~h}} / \mathrm{MIC}$ ratio was $<100$. By increasing the dose to $600 \mathrm{mg}$ once daily, the $\mathrm{AUC}_{0-24 \mathrm{~h}}$ would expectedly increase by about 1.5 -fold [11], resulting in an $\mathrm{AUC}_{0-24 \mathrm{~h}} / \mathrm{MIC}$ ratio $\geqslant 100$. Measuring unbound plasma concentration could obviate the need for dosage adjustment if the unbound $\mathrm{AUC}_{0-24 \mathrm{~h}} / \mathrm{MIC}$ ratio is $>60$, while the total $\mathrm{AUC}_{0-24 \mathrm{~h}} / \mathrm{MIC}$ ratio is $<100$.

We observed a large variability in $\mathrm{AUC}_{0-24 \mathrm{~h}}$ which is unique to this study. The observed variability (nine-fold) could have clinical implications. Based on a median $\mathrm{AUC}_{0-24 \mathrm{~h}}$ of $24.8 \mathrm{mg} \cdot \mathrm{h} \cdot \mathrm{L}^{-1}$ (table 3 ), a standard dose of MFX of $400 \mathrm{mg}$ once daily can be used in the treatment of isolates with a maximum MIC of $0.25 \mathrm{mg} \cdot \mathrm{L}^{-1}$. As both higher MIC values as well as lower $\mathrm{AUC}_{0-24 \mathrm{~h}}$ are measured, the standard dose is not sufficient for all patients. Before increasing the standard dose the $\mathrm{AUC}_{0-24 \mathrm{~h}} / \mathrm{MIC}$ ratio should preferably be assessed by measuring both $\mathrm{AUC}_{0-24 \mathrm{~h}}$ and MIC. Finally, therapeutic drug monitoring (TDM) of MFX was performed in selected patients (i.e. RIF co-medication; MIC $>0.25 \mathrm{mg} \cdot \mathrm{L}^{-1}$ ), and consequently this selection bias could explain the observed variability in MFX AUC $_{0-24 h}$. Nonetheless, the standard dose of $400 \mathrm{mg}$ MFX once daily results in variability in $\mathrm{AUC}_{0-24 \mathrm{~h}}$ values and consequently is probably not sufficient for all patients.

In $66(74.2 \%)$ patients RIF was combined with MFX. However, in accordance with earlier reports [8,9], concomitant treatment with RIF and MFX did cause a decrease of MFX exposure. However, this decrease was not significant. In addition, we observed a nonsignificant increase in apparent clearance in patients with concomitant use of MFX and RIF. This is probably due to a lack of statistical power as full pharmacokinetic curves were not obtained in all patients; besides, intra-group variability in $\mathrm{AUC}_{0-24 \mathrm{~h}}$ in both treatment groups was large. Therefore, our results do not rule out a significant 
drug-drug interaction between RIF and MFX, especially as there was a trend of interaction.

In earlier published work, MFX (400 mg) was well tolerated in 19 TB patients for a period of 180 days [12], in 38 for a period of 174 days [13], in 74 for a period of 56 days [14] and in 53 for a period of 60 days [15]. Less intensive schedules of MFX 3-5 times a week were also well tolerated [16]. Our study with 89 patients with a median treatment of 74 days adds important safety information as our patient population was unselected and therefore represented real life conditions. MFX was well tolerated in our study population; the Naranjo score showed a low probability for the observed adverse effects and MFX was discontinued in only three patients. While first-line anti-TB drugs induced elevated liver enzymes we did not observe any serious adverse events during MFX treatment, in fact, a decrease of liver enzymes was observed. This phenomenon could be due to switching of first-line anti-TB drugs, which induced elevated liver enzymes to MFX. A potential serious but infrequent adverse effect of MFX is the potency to aggravate QT prolongation [11]. Despite several additional risk factors for QT prolongation, no QT prolongation was observed in our population. To prevent treatment failure and suppress resistance against MFX a higher dosage of $600-800 \mathrm{mg}$ will theoretically be needed in most TB patients [7]. In healthy volunteers QT prolongation was observed after administration of $800 \mathrm{mg}$ MFX [11]. However, in these volunteers the observed geometric mean $\mathrm{AUC}_{0-24 \mathrm{~h}}$ value on $800 \mathrm{mg} \mathrm{MFX}$ was $87 \mathrm{mg} \cdot \mathrm{h} \cdot \mathrm{L}^{-1}$, which is 1.8 times the expected $\mathrm{AUC}_{0-24 \mathrm{~h}}$ value of $24.8 \times 2=49.6 \mathrm{mg} \cdot \mathrm{h} \cdot \mathrm{L}^{-1}\left(2 \times \mathrm{AUC}_{0-24 \mathrm{~h}} 400 \mathrm{mg}\right.$ MFX (table 3)) on $800 \mathrm{mg}$ MFX in our TB patients. Taking these results into account, a necessary dose escalation would be safe in most TB patients. However, ECG monitoring is recommended in patients having a high $\mathrm{AUC}_{0-24 \mathrm{~h}}$ and in patients with additional risk factors for QT prolongation [3, 11, 18].

Large variability in plasma protein binding, $\mathrm{AUC}_{0-24 \mathrm{~h}}$ MIC and drug-drug interactions have a large impact on the $\mathrm{AUC}_{0-24 \mathrm{~h}} /$ MIC ratio, and this problem has been incompletely addressed. We assume that $C_{\max }$ or $C_{4}$ may serve as a surrogate predictor for the $\mathrm{AUC}_{0-24 \mathrm{~h}}$ and, consequently, TDM should be possible with limited samples. In patients receiving RIF or in patients infected with isolates for which the MIC of MFX is $\geqslant 0.25 \mathrm{mg} \cdot \mathrm{L}^{-1}$ we recommend measurement of at least a peak MFX level and determination of plasma protein binding, as these cases are at risk for $\mathrm{AUC}_{0-24 \mathrm{~h}} / \mathrm{MIC}$ ratio $<100$. However, in patients suspected of poor absorption due to diarrhoea or vomiting, MFX plasma concentration should be evaluated as well. Patients with MDR-TB may potentially benefit most as the MIC for MFX is usually higher in these patients, but safety of MFX in a dose of $600-800 \mathrm{mg}$ should be carefully monitored.

\section{Conclusion}

MFX treatment was well tolerated in 89 patients, receiving a dose of $400 \mathrm{mg}$ once daily (median dose of $6.7 \mathrm{mg} \cdot \mathrm{kg}^{-1}$ ) for a median duration of 74 days. Evaluation of (un)bound $\mathrm{AUC}_{0-24 \mathrm{~h}} /$ MIC ratio is needed to develop the optimal dosing schedule (fixed or TDM guided) to treat TB patients and prevent resistance.

\section{STATEMENT OF INTEREST}

A statement of interest for the present study can be found at www.erj. ersjournals.com/site/misc/statements.xhtml

\section{ACKNOWLEDGEMENTS}

We thank Bayer (Leverkusen, Germany) for kindly providing the moxifloxacin for our method of analysis.

\section{REFERENCES}

1 van den Boogaard J, Kibiki GS, Kisanga ER, et al. New drugs against tuberculosis: problems, progress, and evaluation of agents in clinical development. Antimicrob Agents Chemother 2009; 53: 849-862.

2 Poissy J, Aubry A, Fernandez C, et al. Should moxifloxacin be used for the treatment of extensively drug-resistant tuberculosis? An answer from a murine model. Antimicrob Agents Chemother 2010; 54: 4765-4771.

3 College ter Beoordeling van Geneesmiddelen. The Medicines Evaluation Board. www.cbg-meb.nl Date last accessed: January 10, 2009. Date last updated: November 30, 2008.

4 Wright DH, Brown GH, Peterson ML, et al. Application of fluoroquinolone pharmacodynamics. J Antimicrob Chemother 2000; 46: 669-683.

5 Shandil RK, Jayaram R, Kaur P, et al. Moxifloxacin, ofloxacin, sparfloxacin, and ciprofloxacin against Mycobacterium tuberculosis: evaluation of in vitro and pharmacodynamic indices that best predict in vivo efficacy. Antimicrob Agents Chemother 2007; 51: 576-582.

6 Peloquin CA, Hadad DJ, Molino LP, et al. Population pharmacokinetics of levofloxacin, gatifloxacin, and moxifloxacin in adults with pulmonary tuberculosis. Antimicrob Agents Chemother 2008; 52: 852-857.

7 Gumbo T, Louie A, Deziel MR, et al. Selection of a moxifloxacin dose that suppresses drug resistance in Mycobacterium tuberculosis, by use of an in vitro pharmacodynamic infection model and mathematical modeling. J Infect Dis 2004; 190: 1642-1651.

8 Nijland HM, Ruslami R, Suroto AJ, et al. Rifampicin reduces plasma concentrations of moxifloxacin in patients with tuberculosis. Clin Infect Dis 2007; 45: 1001-1007.

9 Weiner M, Burman W, Luo CC. Effects of rifampin and multidrug resistance gene polymorphism on concentrations of moxifloxacin. Antimicrob Agents Chemother 2007; 51: 2861-2866.

10 Ball P, Stahlmann R, Kubin R, et al. Safety profile of oral and intravenous moxifloxacin: cumulative data from clinical trials and postmarketing studies. Clin Ther 2004; 26: 940-950.

11 Demolis JL, Kubitza D, Tenneze L, et al. Effect of a single oral dose of moxifloxacin (400 $\mathrm{mg}$ and $800 \mathrm{mg}$ ) on ventricular repolarization in healthy subjects. Clin Pharmacol Ther 2000; 68: 658-666.

12 Valerio G, Bracciale P, Manisco V, et al. Long-term tolerance and effectiveness of moxifloxacin therapy for tuberculosis: preliminary results. J Chemother 2003; 15: 66-70.

13 Codecasa LR, Ferrara G, Ferrarese M, et al. Long-term moxifloxacin in complicated tuberculosis patients with adverse reactions or resistance to first line drugs. Respir Med 2006; 100: 1566-1572.

14 Conde MB, Efron A, Loredo C, et al. Moxifloxacin versus ethambutol in the initial treatment of tuberculosis: a double-blind, randomised, controlled phase II trial. Lancet 2009; 373: 1183-1189.

15 Rustomjee R, Lienhardt C, Kanyok T, et al. A phase II study of the sterilising activities of ofloxacin, gatifloxacin and moxifloxacin in pulmonary tuberculosis. Int J Tuberc Lung Dis 2008; 12: 128-138.

16 Burman WJ, Goldberg S, Johnson JL, et al. Moxifloxacin versus ethambutol in the first 2 months of treatment for pulmonary tuberculosis. Am J Respir Crit Care Med 2006; 174: 331-338.

17 Sacco F, Spezzaferro M, Amitrano M, et al. Efficacy of four different moxifloxacin-based triple therapies for first-line $H$. pylori treatment. Dig Liver Dis 2010; 42: 110-114. 
18 Alffenaar JW, van Altena R, Bokkerink HJ, et al. Pharmacokinetics of moxifloxacin in cerebrospinal fluid and plasma in patients with tuberculous meningitis. Clin Infect Dis 2009; 49: 1080-1082.

19 Stass H, Kubitza D, Schuhly U. Pharmacokinetics, safety and tolerability of moxifloxacin, a novel 8-methoxyfluoroquinolone, after repeated oral administration. Clin Pharmacokinet 2001; 40: Suppl. 1, 1-9.

20 Pranger AD, Alffenaar JW, Wessels AM, et al. Determination of moxifloxacin in human plasma, plasma ultrafiltrate, and cerebrospinal fluid by a rapid and simple liquid chromatographytandem mass spectrometry method. J Anal Toxicol 2010; 34: 135-141.

21 van Klingeren $B$, Dessens-Kroon $\mathrm{M}$, van der Laan $\mathrm{T}$, et al. Drug susceptibility testing of Mycobacterium tuberculosis complex by use of a high-throughput, reproducible, absolute concentration method. J Clin Microbiol 2007; 45: 2662-2668.

22 Nuermberger E, Grosset J. Pharmacokinetic and pharmacodynamic issues in the treatment of mycobacterial infections. Eur $J$ Clin Microbiol Infect Dis 2004; 23: 243-255.
23 Turnidge J. Pharmacokinetics and pharmacodynamics of fluoroquinolones. Drugs 1999; 58: Suppl. 2, 29-36.

24 Proost JH, Eleveld DJ. Performance of an iterative two-stage bayesian technique for population pharmacokinetic analysis of rich data sets. Pharm Res 2006; 23: 2748-2759.

25 U.S. Department of Health and Human Services. Common Terminology Criteria for Adverse Events (CTCAE) version 4.0. $\mathrm{NIH}$ publication no. 09-5410. Bethesda, NIH, 2010. Available from: http:/ /evs.nci.nih.gov/ftp1/CTCAE/CTCAE_4.03_2010-06-14_Quick Reference_8.5x11.pdf.

26 Zareba W, Cygankiewicz I. Long QT syndrome and short QT syndrome. Prog Cardiovasc Dis 2008; 51: 264-278.

27 Zemrak WR, Kenna GA. Association of antipsychotic and antidepressant drugs with Q-T interval prolongation. Am J Health Syst Pharm 2008; 65: 1029-1038.

28 Viskin S, Justo D, Halkin A, et al. Long QT syndrome caused by noncardiac drugs. Prog Cardiovasc Dis 2003; 45: 415-427.

29 Naranjo CA, Busto U, Sellers EM, et al. A method for estimating the probability of adverse drug reactions. Clin Pharmacol Ther 1981; 30: 239-245. 